\title{
Response by the authors
}

\author{
Tobias Kelly, PhD*, Steffen Jensen, PhD**, Morten Koch Andersen, PhD**, Catrine \\ Christiansen, PhD*** , Jeevan Raj Sharma PhD*
}

We would like to thank the editors and the commentators for their comments and the opportunity to respond. We greatly appreciate the time and effort that such experienced and expert practitioners have taken to read our paper.

We think the Istanbul Protocol is a crucially important document in the struggle against torture, and it is therefore very important to think about the ways in which it is used in practice as part of a wider campaign to prevent torture and provide survivors with redress. More broadly, our general aim is to participate in a wider debate about the ways in which human rights organizations can produce effective evidence in the struggle to end impunity and provide justice.

We welcome this opportunity to clear up some misunderstandings that may have arisen.

We do not say in the article that the IP requires comprehensive documentation. Indeed we say early on in the paper that it is designed as a flexible protocol. It is all the more important therefore to think about how the IP can be best used in practice, given the range of potential options it presents. The article therefore sets out to see if, how and when human rights practitioners use the IP in the context of wider human rights processes. Our conclusion is not that the IP

\footnotetext{
*) School of Social and Political Science, University of Edinburgh, Scotland

$\left.{ }^{\star \star}\right)$ Dignity: Danish Institute Against Torture,

Copenhagen, Denmark.

$\star \star \star$ Independent consultant
}

is inapplicable to Low-Income Countries, but the much more limited claim that more comprehensive forms of forensic documentation, as set out in the IP, will be limited to a small, albeit, important number of cases.

The commentators are concerned that we have included the opinions of non-clinicians in our study. We did this deliberately. Our aim was to examine how documentation is used as part of human rights work. Journalists and lawyers are certainly not qualified to carry out forensic documentation. However, they are often the ones who use such documentation as part of wider attempts to seek justice. To end the discussion of the IP at what clinicians think would be to ignore the ways in which the IP should be seen as a tool in these broader struggles.

The three countries in which we carried out the study can all be classified as LowIncome. We do not make any causal inferences about the ways in which Low-Income status impacts on human rights work. We of course recognize that impunity is a problem all over the world. At the same time, it is also important to recognize that resource limitations are more acute in some places than in others.

We are certainly not suggesting that human rights aspirations should be low in Low-Income countries, and say so explicitly in the introduction. We do think though that human rights struggles, at a tactical level, do not operate in a vacuum and they therefore need to take the political and legal context into account; it is only by doing so that they can be effective. 